Research Article

\title{
Reliability Assessment for Very Few Failure Data and Weibull Distribution
}

\author{
Lulu Zhang ${ }^{D}$, Guang Jin, and Yang You \\ College of Systems Engineering, National University of Defense Technology, Changsha 410073, China \\ Correspondence should be addressed to Lulu Zhang; shuyin123@163.com
}

Received 3 July 2019; Revised 16 August 2019; Accepted 5 September 2019; Published 25 September 2019

Academic Editor: Mijia Yang

Copyright ( $\odot 2019$ Lulu Zhang et al. This is an open access article distributed under the Creative Commons Attribution License, which permits unrestricted use, distribution, and reproduction in any medium, provided the original work is properly cited.

\begin{abstract}
Only very few failure data can be obtained for the time censored test of high-reliability and long-life products. For very few failure data, the current methods fail to obtain both the point estimation and confidence interval of reliability parameters. If the point estimation and confidence interval of reliability parameters are obtained based on different methods, the results tend to be unreliable. In this study, based on the existing research, a Bayesian reliability evaluation method for very few failure data under the Weibull distribution was proposed. First, the range of failure probability was limited based on the convexity and self-features of the Weibull distribution function. Second, based on the background of the sample with very few failure data, the pretest distribution function and parameters were set and solved. The point estimation and confidence interval model of failure probability based on the Bayesian formula was established. The improved match distribution curve method was used to compute both the point estimation and confidence interval of reliability parameters. Furthermore, by comparing the results of numerical examples, the calculation results obtained by the proposed method were verified as being very reasonable. Finally, taking wet friction plates as an example, the results showed the effectiveness of this method in engineering practice.
\end{abstract}

\section{Introduction}

In product design, research and development, production, improvement, and other aspects, it is usually necessary to verify the reliability of the product through reliability tests. For current products, the reliability is high and the life is longer and longer. The failure data collected by the reliability test have become less and less, and even sometimes the failure data cannot be collected. How to study the reliability of the product under the condition of very few failure data has become an urgent problem that needs to be solved.

The Bayesian method is an effective method to deal with the reliability problem of products with few failure information. It has been widely used in reliability parameter point estimation [1, 2]. Martz and Waller [3] studied the reliability evaluation problem under the condition of no failure data for the first time. After decades of development, research on this issue has achieved a lot of findings $[4,5]$. Kurz et al. [6] studied the early life failure of a product and established the advanced Bayesian estimation models under the Weibull distribution. Jiang et al. [7] based on the different values of the shape parameters in the Weibull distribution found that the value of the probability of failure was determined by the characteristics of the concavity and distribution function, and the point estimation value of the failure probability was calculated. Yi et al. [8] proposed an E-Bayesian reliability evaluation method based on the condition of exponential distribution and zero-failure data, and the method was applied to the analysis of the seekers failure rate and reliability. In order to solve the problem that the Weibull parameter prior distribution is difficult to determine, Kan et al. [9] introduced the expert judgment process and established the multiple numerical control machine tools (NCMTs) Bayesian failure-free data reliability model and evaluation method. The above method studies the reliability parameter point estimation of products without failure data but did not discuss the confidence interval of reliability parameters.

With regard to the confidence interval problem of reliability parameters under the condition of no failure data, 
the optimal confidence limit method was proposed [10] and the point estimation of reliability parameters and the oneside confidence lower limit of parameters were obtained. Li et al. [11] proposed a new model to solve the consistency problem, which improved the accuracy of reliability interval estimation based on the reliability evaluation. The product reliability problem under unequal time censored failure data was studied [12]. The time censored test was extended, and the solution method of the lower confidence limit under the Weibull distribution and exponential distribution was given. $\mathrm{Xu}$ and chen [13] proposed the method of the two-sided modified Bayesian (M-Bayesian) credible limit and used this method to study the failure probability and reliability confidence interval evaluation method under the condition of no failure data. It can be seen from the current research that the use of the distribution curve method is often limited to the point estimation of the parameters. It is rarely used in the parameter confidence interval solving process. In addition, the reliability of the product under the condition of no failure data has been studied more. However, it is rare to find a reliability assessment method for very few failure data conditions. The traditional reliability assessment method is generally based on obtaining more failure data, and the traditional method is often used in the case of very few failure data, resulting in a large deviation.

In the Weibull distribution, the match distribution curve method was improved for the characteristics of very few failure data, and the range of failure probability was limited according to the value of the shape parameters $m$ and thus further improved the accuracy of the evaluation range. This method can analyze the reliability evaluation of products under the condition of few failure data, including the point estimation of parameters and the confidence interval estimation of parameters and fundamentally solve "disjointed" problems between parameter point estimation and parameter interval estimation.

\section{Weibull Distribution}

In the reliability evaluation of products, the first step is to assume the probability distribution form of product life compliance, on the basis of which the sample information is used to evaluate the distribution parameters, and further calculate the reliability indicators. Life distribution is the basis of reliability research. After years of development, the commonly used life distributions include exponential distribution, gamma distribution, Weibull distribution, and lognormal distribution, where the Weibull distribution is derived from the weakest link model. This model is like a chain in which many rings are connected in series. When both ends are subjected to tension, any one of the rings can break, the chain will fail, and the chain failure will occur in the most vulnerable link. Therefore, the life of units, components, devices, and equipment which are not working properly due to partial failure can be regarded as approximating the Weibull distribution. Most electronic products, mechanical products, and electrical products (such as bearings, generators, hydraulic pumps, and materials) are subjected to the Weibull distribution [14].
The Weibull distribution is defined as

$$
f(t)=\frac{m}{\eta}\left(\frac{t}{\eta}\right)^{m-1} \exp \left[-\left(\frac{t}{\eta}\right)^{m}\right], \quad t>0,
$$

where $T \sim W(m, \eta), m$ is shape parameters, and $\eta$ is scale parameters.

The cumulative distribution function (CDF) of Weibull distribution is defined as

$$
F(t)=P(T \leq t)=1-\exp \left[-\left(\frac{t}{\eta}\right)^{m}\right] .
$$

For $R(t)=1-F(t)$,

$$
R(t)=\exp \left[-\left(\frac{t}{\eta}\right)^{m}\right]
$$

Combined with the reliability formula $\lambda(t)=$ $(f(t) / R(t))$, the failure rate function under the Weibull distribution is $\lambda(t)=(m / \eta)(t / \eta)^{m-1}$. When the shape parameter $m<1$, the failure probability density function $f(t)$ and the failure rate function $\lambda(t)$ are both decreasing functions, which describe sudden failure, which is equivalent to the early failure of the product. When $m=1$, the Weibull distribution is the exponential distribution. When $m=2$, the Weibull distribution is the Rayleigh distribution. When $m>3$, the Weibull failure probability density function has single-peak symmetry, which approximates a normal distribution and describes the product gradual failure. At the same time, it indicates the combination of sudden failure and gradual failure, in which $m$ can be adjusted according to different failure types.

\section{Model}

In the reliability test of products with higher reliability, test results often show a situation in which very few test samples fail during the entire reliability test or even no sample failures, and the number of such failed samples is less than $10 \%$ (including when the number of failed samples is 0 ), and this is called the minimum data failure test. Reliability research work based on this is important for predicting the life of highly reliable products.

In the product life comprehensive test verification method, the truncation life test is divided into two categories, one is the fixed censored test and the other is the time censored test. Of them, the time censored test needs to collect more test data in order to evaluate the reliability of the product with time and is used for products with a short life cycle and a low test cost. In reality, the problem of collecting only very few failure data occurs mainly in the time censored test. This study evaluates product reliability based on the minimum failure data obtained from the time censored test.

Assume there are $N$ samples in the time censored test, and a total of $k(k \leq N)$ time censored tests are performed. The end of each time censored test $\tau_{i}(i=1,2, \ldots, k)$ is indicated. There are $n_{i}$ samples observed at each censored test time $\tau_{i}(i=1,2, \ldots, k)$ and $\sum_{i=1}^{k} n_{i}=N$. If there are failed samples in the period of $\left(\tau_{i-1}, \tau_{i}\right]$, the failure time 
$t_{i j}\left(j=1,2, \ldots, r_{i}\right)$ is recorded corresponding to the number of failed samples $r_{i}$. The samples are numbered after each time, from 1 to $N$. After all the time censored tests are completed, the life data $t_{i}(i=1,2, \ldots, N)$ are collected corresponding to the sample number. The special case is that the failure data are not collected at this time, they are a special case where there are very little failure data.

In order to facilitate the subsequent derivation, let $s_{i}=\sum_{j=i}^{k} n_{j}, s_{i}$ indicates the number of samples still participating at time $\tau_{i}(i=1,2, \ldots, k)$ in the time censored test plus the number of internal $\left(\tau_{i-1}, \tau_{i}\right]$ failed samples $r_{i}$. In addition, the probability of failure at each truncation moment is recorded $p_{i}=F\left(\tau_{i}\right)=P\left(t \leq \tau_{i}\right), i=1,2, \ldots, k$, and it is known that $p_{1} \leq p_{2} \leq \cdots \leq p_{k}$ based on the test results.

This study discussed the solution method of $R(t)$ based on the match distribution curve method. First, it is necessary to estimate the failure probability $p_{i}$ of each truncation moment $\tau_{i}$ and fit the distribution curve based on $\left(\tau_{i}, p_{i}\right)$, and then further estimate other required distribution parameters, wherein the failure probability is the key to the method.

\section{Failure Probability Estimation}

For the estimation of $p_{i}$, the classical estimation method used in the past [15] was $p_{i}=\left(r_{i}+0.5 / s_{i}+1\right)$, if there is no sample failure within the time period $\left(\tau_{i-1}, \tau_{i}\right]$, $p_{i}=\left(0.5 / s_{i}+1\right)$. This failure probability estimation method is too rough, and the calculation accuracy is poor. In order to overcome the problem of poor calculation accuracy of the classical method, $p_{i}$ is set as a random variable and the Bayesian method $[16,17]$ is used to estimate the poster distribution of $p_{i}$

$$
\pi\left(p_{i} \mid t_{1}, \ldots, t_{N}\right)=\frac{\pi\left(p_{i}\right) L\left(t_{1}, \ldots, t_{N} \mid p_{i}\right)}{\int_{p_{i}} \pi\left(p_{i}\right) L\left(t_{1}, \ldots, t_{N} \mid p_{i}\right) d p_{i}},
$$

where $t_{1}, \ldots, t_{N}$ is the sample information of the samples, $L\left(t_{1}, \ldots, t_{N} \mid p_{i}\right)$ is the likelihood function of the sample, and $\pi\left(p_{i}\right)$ is the prior distribution. It can be seen from the above formula that the Bayesian method can fuse a variety of information, which can further improve the estimation accuracy of the failure probability $p_{i}$. There are two key problems that need to be solved by the Bayesian formula. First, it is necessary to determine the range of values of the failure probability $p_{i}$. Second, it is necessary to determine the distribution form (i.e., distribution function) and distribution parameters of the prior distribution.

4.1. Range of Failure Probability. The value range of the failure probability $p_{i}$ is $\left[l_{i}, u_{i}\right]$, and generally the value range of the failure probability $p_{i}$ is assumed to be $[0,1]$. This assumption of the failure probability is too conservative, and the calculation accuracy is poor. In order to improve the accuracy of the range of failure probability, for the characteristics of the failure probability range of the product with very few failure data, this study used the concavity and function of the distribution function to set the range of failure probability.
By taking the second derivative of the Weibull distribution function (2), we have

$$
\begin{aligned}
\frac{d^{2} F(t)}{d t^{2}} & =\frac{m t^{m-2} \exp \left(-(t / \eta)^{m}\right)}{\eta^{m}}\left(m-1-\frac{m t^{m}}{\eta^{m}}\right) \\
& =\frac{m^{2}}{\eta^{2}}\left(\frac{t}{\eta}\right)^{m-2}\left(\frac{m-1}{m}-\frac{t^{m}}{\eta^{m}}\right) \exp \left[-\left(\frac{t}{\eta}\right)^{m}\right] .
\end{aligned}
$$

When $m \leq 1$, it is easy find from (5) that $\left(d^{2} F(t) / d t^{2}\right)<0$ and the distribution function $F(t)$ is a convex function, and according to the nature of the convex function, we have

$$
\frac{p_{1}}{t_{1}}>\frac{p_{2}}{t_{2}}>\cdots>\frac{p_{i-1}}{t_{i-1}}>\frac{p_{i}}{t_{i}}>\cdots>\frac{p_{k}}{t_{k}} .
$$

Combining $p_{1} \leq p_{2} \leq \cdots \leq p_{k}$, the interval of $p_{i}$ is

$$
p_{i-1} \leq p_{i}<\frac{t_{i}}{t_{i-1}} p_{i-1}, \quad i \geq 2 .
$$

First, it is necessary to define the value range $0<p_{1}<p_{\text {lim }}$ of $p_{1}$, where $p_{\text {lim }}$ is the upper limit of $p_{1}$. The value setting of $p_{\lim }$ is based on the actual situation of the sample and expert experience. The value is generally not more than 0.5 in actual engineering.

When $m>1$, it is difficult to determine the concavity and convexity of the distribution function. Based on the function characteristics, (2) is transformed into the following equation:

$$
\ln \ln \frac{1}{1-p}=m \ln t-m \ln \eta
$$

Putting $p_{i-1}$ and $p_{i}$ into (8), it results in the following equation:

$$
\ln \frac{\ln \left(1-p_{i-1}\right)}{\ln \left(1-p_{i}\right)}=m \ln \frac{t_{i-1}}{t_{i}}<\ln \frac{t_{i-1}}{t_{i}}
$$

Then, (9) is transformed into

$$
\frac{\ln \left(1-p_{i-1}\right)}{\ln \left(1-p_{i}\right)}=m \frac{t_{i-1}}{t_{i}}>\frac{t_{i-1}}{t_{i}} .
$$

Then, $p_{i}>1-\left(1-p_{i-1}\right)^{t_{i} / t_{i-1}}$, and the lower limit of $p_{i}$ is $1-\left(1-p_{i-1}\right)^{t_{i} / t_{i-1}}$. Let the upper limit of $p_{i}$ be $p_{\text {upp }}$, and the value of $p_{\text {upp }}$ is based on the actual situation. Based on the distribution function and the function characteristics, the value interval of $p_{i}$ is defined as

$$
\begin{aligned}
& l_{i} \begin{cases}p_{i-1}, & m \leq 1, \\
1-\left(1-p_{i-1}\right)^{t_{i} / t_{i-1}}, & m>1,\end{cases} \\
& u_{i} \begin{cases}\frac{t_{i}}{t_{i-1}} p_{i-1}, & m \leq 1, \\
p_{\text {upp }}, & m>1 .\end{cases}
\end{aligned}
$$

Since the exact value of the failure probability $p_{i}$ cannot be obtained during the calculation, the estimated value $\widehat{p}_{i}$ is used to replace failure probability $p_{i}$. 
4.2. Prior Distribution Form and Distribution Parameters. From the sample data and historical data, the prior distribution is a nonuniform distribution, which is closer to the beta distribution. In addition, the beta distribution Beta ( $a$, $b$ ) is also the conjugate prior distribution of the parameters, and the conjugate prior distribution can be easily integrated from the historical information. It provides a reasonable premise for the analysis of future experimental data, and some parameters of its posterior distribution can also be well explained [18]; this study used Beta $(a, b)$ as the prior distribution. According to the change of failure probability value range, the value range of $\operatorname{Beta}(a, b)$ is adjusted accordingly:

$$
\pi\left(p_{i} \mid a, b\right)=\frac{p_{i}^{a-1}\left(1-p_{i}\right)^{b-1}}{B_{i}(a, b)}
$$

where $B_{i}(a, b)=\int_{l_{i}}^{u_{i}} x^{a-1}(1-x)^{b-1} d x$.

Since the reliability and data collected in the time censored test are very small, the failure probability $p_{i}$ is small and the possibility of a large value is small. This is consistent with the characteristics of the subtraction function, and the prior distribution density function $\pi\left(p_{i} \mid a, b\right)$ is derived:

$$
\begin{aligned}
\frac{d \pi\left(p_{i} \mid a, b\right)}{d p_{i}}= & \frac{p_{i}^{a-2}\left(1-p_{i}\right)^{b-2}}{B_{i}(a, b)} \\
& \cdot\left[(a-b)\left(1-p_{i}\right)-p_{i}(b-1)\right] \leq 0 .
\end{aligned}
$$

Equation (13) is only true when $a \in(0,1]$ and $b \geq 1$. According to the nature of the probability density of the Bates distribution function, when $a \in(0,1]$, the larger the $b$, the finer the tail of the probability density function. From the perspective of Bayesian estimation of robustness, a finer right tail of the prior distribution makes the Bayesian estimation less robust, so the value must have an upper limit. It is $c$. In practical applications, $c$ should be selected in $[2,8]$, and the arbitrary value in the interval $[2,8]$ has little influence on the reliability, failure probability, and other indicators, which can be ignored [18]. It is very difficult to further determine the exact values of $a$ and $b$. Rather than using expert experience to determine a uniquely determined value, as it is not as uniform as the hypothesis and within the respective range of $a$ and $b$, we have

$$
\left\{\begin{array}{l}
\pi_{i}(a)=U(0,1) \\
\pi_{i}(b)=U(0, c) .
\end{array}\right.
$$

The prior distribution of $p_{i}$ is

$$
\pi\left(p_{i}\right)=\int_{1}^{c} \int_{0}^{1} \frac{p_{i}^{a-1}\left(1-p_{i}\right)^{b-1}}{(c-1) B(a, b)} d a d b .
$$

\subsection{Bayesian Failure Probability Estimation}

4.3.1. Failure Probability Estimation without Zero-Failure Data. The failure probability acquisition under the condition of zero-failure data was studied [7]. In the time-censored test, there are $s_{i}$ test samples at time $\tau_{i}$. The likelihood function of $p_{i}$ is $L\left(s_{i} \mid p_{i}\right)=\left(1-p_{i}\right)^{s_{i}}$. Let $\left(1-p_{i}\right)^{2}$ denote he core of the prior distribution. To meet the requirement of prior distribution $\int_{p} A\left(1-p_{i}\right)^{2} d p=1$, the prior distribution function is $\pi\left(p_{i}\right)=\left(3\left(1-p_{i}\right)^{2} /\left(\left(1-p_{l}\right)^{3}-\left(1-p_{u}\right)^{3}\right)\right)$. Based on the Bayesian formula (4), the poster distribution can be found:

$$
\pi\left(p_{i} \mid s_{i}\right)=\frac{\left(s_{i}+3\right)\left(1-p_{i}\right)^{s_{i}+2}}{\left(1-p_{l}\right)^{s_{i}+3}-\left(1-p_{u}\right)^{s_{i}+3}}
$$

Under the square loss assumption, the expectation of $p_{i}$ is

$$
\left\{\begin{array}{l}
\widehat{p}_{i}=\frac{s_{i}+3}{\left(1-p_{l}\right)^{s_{i}+3}-\left(1-p_{u}\right)^{s_{i}+3}}\left[q\left(p_{u}\right)-q\left(p_{l}\right)\right], \\
q(x)=\frac{(1-x)^{s_{i}+4}}{s_{i}+4}-\frac{(1-x)^{s_{i}+3}}{s_{i}+3} .
\end{array}\right.
$$

4.3.2. Failure Probability Estimation Based on Very Few Failure Data. From the time censored test, there are $s_{i}$ samples remaining in the test at censoring time $\tau_{i}$, where $r_{i}$ samples have failed. The likelihood function of $p_{i}$ is

$$
L\left(s_{i}, r_{i} \mid p_{i}\right)=C_{s_{i}}^{r_{i}} p_{i}^{r_{i}}\left(1-p_{i}\right)^{s_{i}-r_{i}} .
$$

Putting (15) and (18) into Bayesian formula (4), we can obtain the poster distribution:

$$
\pi\left(p_{i} \mid s_{i}, r_{i}\right)=\frac{\int_{1}^{c} \int_{0}^{1}\left(p_{i}^{a+r_{i}-1}\left(1-p_{i}\right)^{s_{i}-r_{i}+b-1} / B_{i}(a, b)\right) d a d b}{\int_{1}^{c} \int_{0}^{1} \int_{l_{i}}^{u_{i}}\left(p_{i}^{a+r_{i}-1}\left(1-p_{i}\right)^{s_{i}-r_{i}+b-1} / B_{i}(a, b)\right) d p_{i} d a d b},
$$

where $p_{i} \in\left[l_{i}, u_{i}\right]$ is the value obtained from (11). Under the square loss assumption, the poster distribution expected value of Bayesian poster point estimation $\widehat{p}_{i}$ is

$$
\begin{aligned}
\widehat{p}_{i} & =\int_{l_{i}}^{u_{i}} p_{i} \pi\left(p_{i} \mid s_{i}, r_{i}\right) d p_{i} \\
& =\frac{\int_{1}^{c} \int_{0}^{1} \int_{l_{i}}^{u_{i}}\left(p_{i}^{a+r_{i}}\left(1-p_{i}\right)^{s_{i}-r_{i}+b-1} / B_{i}(a, b)\right) d p_{i} d a d b}{\int_{1}^{c} \int_{0}^{1} \int_{l_{i}}^{u_{i}}\left(p_{i}^{a+r_{i}-1}\left(1-p_{i}\right)^{s_{i}-r_{i}+b-1} / B_{i}(a, b)\right) d p_{i} d a d b}
\end{aligned}
$$

where the integral calculation can be performed by the numerical integration method.

\section{Reliability Estimation Based on Very Few Failure Data}

After obtaining the point estimation $\widehat{p}_{i}$ of failure probability $p_{i}$, the distribution curve method can be used to fit the points into a distribution curve. In this study, the weighted least squares estimation method was used to fit the distribution curve.

Let $y=\ln [-\ln (1-p)], x=\ln t, b=m \ln \eta$, and then (2) is transformed into 


$$
y=m x-b
$$

The weighted least squares estimation method is used to fit $\left(x_{i}, \widehat{y}_{i}\right)$, obtaining the point estimation of parameters $m$ and $\eta$, and then the reliability is estimated. By minimizing the square of fitting errors, we have

$$
Q=\sum_{i=1}^{k} w_{i}\left(\hat{y}_{i}-m x_{i}+m \ln \eta\right)^{2},
$$

where $w_{i}=\left(n_{i} t_{i} / \sum_{j=1}^{k} n_{j} t_{j}\right), k \geq 2$.

Let $A=\sum_{i=1}^{k} w_{i} x_{i}, B=\sum_{i=1}^{k} w_{i} x_{i}^{2}, C=\sum_{i=1}^{k} w_{i} y_{i}$, and $D=$ $\sum_{i=1}^{k} w_{i} x_{i} y_{i}$, and with the derivative calculation of (22), we obtain

$$
\begin{aligned}
& \widehat{m}=\frac{D-A C}{B-A^{2}}, \\
& \widehat{\eta}=\exp \left(\frac{A D-B C}{D-A C}\right) .
\end{aligned}
$$

The estimated distribution parameters obtained from (23) are put into (3), getting the estimation $\widehat{R}(t)$ based on the Weibull distribution:

$$
\widehat{R}(t)=R(t ; \widehat{m}, \widehat{\eta})=\exp \left[-\left(\frac{t}{\widehat{\eta}}\right)^{\widehat{m}}\right] .
$$

\section{Confidence Interval Estimation Based on Very Few Failure Data}

For the Weibull confidence interval, Han [19] proposed the calculation method of the optimal confidence lower bound under the condition of no failure data and then obtained the lower confidence limit of the Weibull distribution under the confidence level $(1-\alpha)$ :

$$
R_{Z}^{L}(t)=\exp \left(\frac{t^{m} \ln \alpha}{\sum_{i=1}^{k} n_{i} \tau_{i}^{m}}\right),
$$

where the shape parameter $m$ is the real value, but this is difficult to obtain in the actual use process, and therefore, the point estimation value $\widehat{m}$ is generally used instead, which will bring a certain error into the final calculation result. In addition, the confidence interval estimation is different from the method used for point estimation, and it is easy to have a "disjointed" problem. If the acquired information is used, the Weibull partitioning interval is obtained based on the distribution curve method, and the secondary error can be further avoided.

Under confidence level $(1-\alpha)$, first the upper bound $\hat{p}_{i}^{u}$ of the failure probability $p_{i}$ is estimated. Based on the poster distribution in (19), $G(x, s, r)$ is defined as

$$
G(x, s, r)=\int_{l}^{x} \frac{\int_{1}^{c} \int_{0}^{1}\left(\left(p^{a+r-1}(1-p)^{s-r+b-1} / B_{i}(a, b)\right) d a d b\right)}{\int_{1}^{c} \int_{0}^{1} \int_{1}^{u}\left(\left(p^{a+r-1}(1-p)^{s-r+b-1} / B_{i}(a, b)\right) d p d a d b\right)} d p .
$$

According to the definition of the upper confidence limit, we have

$$
G\left(\widehat{p}_{i}^{u}, s_{i}, r_{i}\right)=1-\alpha .
$$

It is known that $G\left(l_{i}, s_{i}, r_{i}\right)=0, G\left(u_{i}, s_{i}, r_{i}\right)=1$. Equation (27) is solved by dichotomy, and the upper confidence limit $\widehat{p}_{i}^{u}$ of failure probability $p_{i}$ is obtained. Based on the linearization process of (2), we have $\hat{y}_{i}^{u}=\ln \left[-\ln \left(1-\widehat{p}_{i}^{u}\right)\right]$ and $x_{i}=\ln t_{i}$. According to the weighted least squares method, the upper limit curve of the failure probability is obtained by fitting points $\left(x_{i}, \widehat{y}_{i}^{u}\right)$. From (22), we have

$$
Q^{u}=\sum_{i=1}^{k} w_{i}\left(\hat{y}_{i}^{u}-m x_{i}+m \ln \eta\right)^{2} .
$$

Because the value of $m$ is limited and the value has little effect on the reliability index value, the confidence interval of $m$ is not assumed here. The point estimation value of $m$ is $\widehat{m}$. After obtaining the point estimation $\widehat{m}$, the lower confidence limit $\eta_{L}$ of the estimation value $\eta$ is obtained. Putting $\widehat{m}$ into (28), we have

$$
Q^{u}=\sum_{i=1}^{k} w_{i}\left(\widehat{y}_{i}^{u}-\widehat{m} x_{i}+\widehat{m} \ln \eta\right)^{2} .
$$

The lower confidence limit $\eta_{L}$ can be obtained when the value of $Q^{u}$ is the smallest:

$$
\eta_{L}=\exp \left[\sum_{i=1}^{k} w_{i}\left(x_{i}-\frac{y_{i}^{u}}{\widehat{m}}\right)\right] \text {. }
$$

The point estimation value $\widehat{m}$ and the lower confidence limit $\eta_{L}$ are added to the Weibull reliability calculation formula, and then the lower confidence limit $R$ of the reliability at the confidence level $(1-\alpha)$ can be obtained at any time $t$ :

$$
R_{L}(t)=\exp \left[-\left(\frac{t}{\eta_{L}}\right)^{\widehat{m}}\right]
$$

Similarly, the upper confidence limit for the probability of failure is

$$
F_{u}(t)=1-R_{L}(t)=1-\exp \left[-\left(\frac{t}{\eta_{L}}\right)^{\widehat{m}}\right] .
$$

The upper confidence limit of the failure rate function is

$$
\lambda_{u}(t)=\frac{f_{u}(t)}{R_{L}(t)}=\frac{\widehat{m}}{\eta_{L}}\left(\frac{t}{\eta_{L}}\right)^{\widehat{m}-1} .
$$

\section{Simulation Verification}

In order to verify the accuracy of the proposed method, experiments were used to generate simulation samples. Based on the generated simulation samples, the classical estimation method [15], the zero-failure estimation method [7], and the very few failure data evaluation method proposed in this study were used to evaluate the reliability of the samples and compare the evaluation result with the true value. The simulation experiment was divided into two cases, 
one was the simulation experiment without failure data and the other was the simulation experiment under the condition of very few failure data. The generation process of the simulation sample was described by Zhang et al. [20].

(1) Generation of simulation samples with zero-failure data:

(a) Set the true value of the shape parameter $m$ and the scale parameter $\eta$ in the Weibull distribution, and generate the failure time $t_{i}(i=1,2, \ldots, N)$ through MATLAB, that is, generate a total of $N$ experimental samples in the simulation experiment

(b) Generate $N$ uniformly distributed random numbers $u_{i}, u_{i} \in(0,1)$

(c) $t_{i}$ is replaced by $u_{i} t_{i}$ to indicate no-failure truncation time $t_{i}$

(d) According to the value range of no-failure time $t_{i}, t_{i}$ is divided into $k$ group censored experiments. The ending data $n_{j}$ of each group is taken as the ending data, and the minimum value in the truncation data is taken as the truncation time, which constitutes the sample size $\left(t_{j}, n_{j}\right), j=$ $1,2, \ldots, k$

(2) The generation of simulation samples under very few failure data conditions:

(a) Set the true value of the shape parameter $m$ and the scale parameter $\eta$ in the Weibull distribution, and generate the failure time $t_{i}(i=1,2, \ldots, N)$ through MATLAB, that is, generate a total of $N$ experimental samples in the simulation experiment

(b) Generate $N$ uniformly distributed random numbers $u_{i}, u_{i} \in(0,1)$

(c) Determine the censored level (CL), and on this basis, arbitrarily select $r(r=N \times C L)$ and the expiration time $t_{f i}(i=1,2, \ldots, r)$ from all, denoted as $t_{f i}(i=1,2, \ldots, r)$, and the remaining expiration time $t_{s i}(i=1,2, \ldots, N-r)$ is used

(d) $t_{i}$ is replaced by $u_{i} t_{i}$ to indicate no-failure truncation time $t_{i}$

(e) According to the value of $t_{f i}(i=1,2, \ldots, r)$ and $t_{s j}$, the truncation time is grouped and the minimum truncation time is taken as the truncation time of the censored experiment of the group

It was assumed that 10 samples will be selected for 5 censored experiments. The shape parameters $m=2$ and scale parameters $\eta=2500$ in the Weibull distribution are known. The simulation samples were generated under the condition of zero-failure data, as shown in Table 1.

According to the time censored test data in Table 1, Figure 1 shows the point estimation value of the failure probability solved by the classical estimation method [15], the zero-failure estimation method [7], and the very few failure data reliability evaluation method proposed in this study. The absolute error with the true value is shown in Figure 2.
TABle 1: Simulation sample data.

\begin{tabular}{lcc}
\hline Number & Censoring time $(\mathrm{h})$ & Number of samples \\
\hline 1 & 435.357 & 1 \\
2 & 938.124 & 1 \\
3 & 1263.982 & 1 \\
4 & 1517.114 & 2 \\
5 & 1735.365 & 5 \\
\hline
\end{tabular}

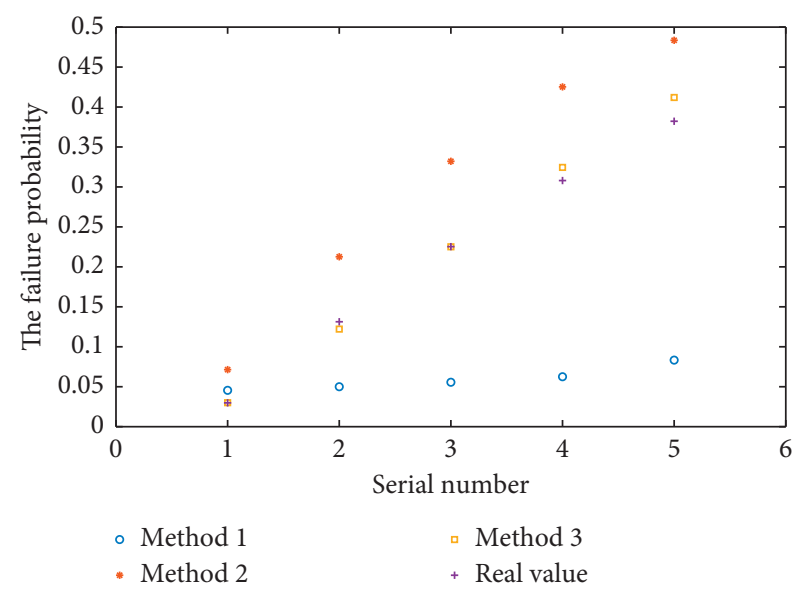

FIGURE 1: Comparison of the failure probability point estimation.

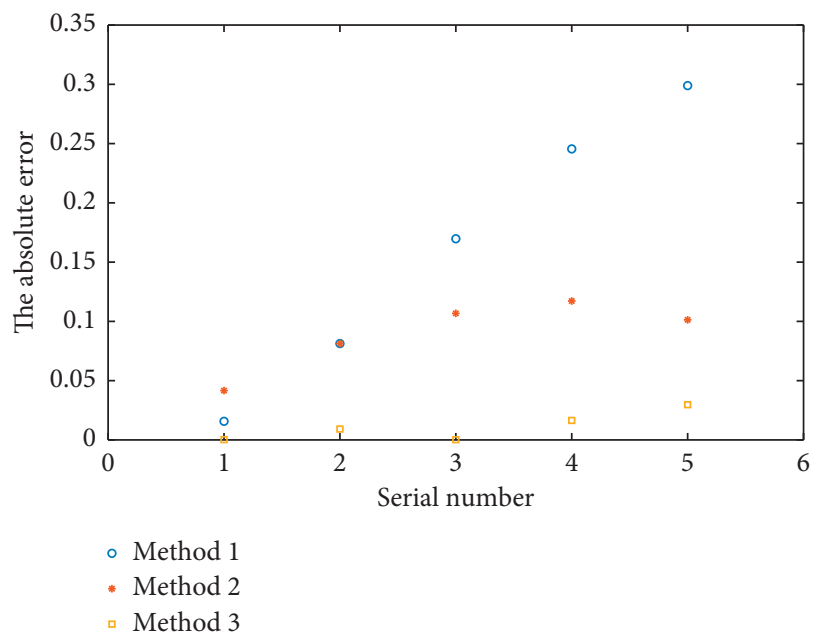

FIgURE 2: Comparison of the absolute error of the failure probability.

In the figure, Method 1 is the classical estimation method [15], Method 2 is the no-failure estimation method [7], and Method 3 is the minimum data failure reliability evaluation method proposed in this study. It can be seen from Figures 1 and 2 that the minimum data failure reliability assessment method can be used to estimate the failure probability of nonfailure data sample products, and the estimation result is closest to the true value. The classical estimation method [15] does not consider the truncation time versus failure in the time-censored test. The influence of probability makes the probability of failure "over-underestimated," while the method of zero-failure estimation [7] has certain 
randomness in the process of determining the kernel of the prior distribution of failure probability, making the estimation probability of failure probability "large."

As Jia et al. [15] and Jiang et al. [7] did not further analyze the confidence interval of the reliability parameter based on the estimation of the failure probability point, in order to facilitate the comparison, the confidence level was obtained by the optimal confidence limit method of equation (25). Under confidence level 0.9 , the corresponding reliability lower confidence limit (LFM), the comparison results are shown in Figure 3.

Comparing Figure 1 with Figure 3, it can be seen that the lower confidence limit of reliability is calculated by using the optimal confidence limit method on the basis of the estimation results of failure probability points obtained by the classical estimation methods, namely, Method 1 [15] and Method 2 [7]. Compared with the estimation results of parameter points, there is a distinct "disconnection" problem, and the calculation results of the lower confidence limit are not credible. Method 3 proposed in this study effectively overcomes the problem of "disconnection" in the process of obtaining the lower confidence limit, and the calculation results are more reasonable and more reliable.

\section{Case Study}

Clutches in integrated transmissions are mostly hydraulically controlled wet multiplate clutches. This clutch allows the shifting process to be gentle, making it easy to shift power and automate the shifting process. The wet multiplate shifting clutch is composed of many annular double-sided steel sheets and friction plates, and the dual steel sheets and the friction plates are arranged between the two and can be used as an active sheet or a passive sheet. The friction sheet is generally used as an active sheet. The active piece is connected to the spline on the input shaft by an internal spline, and the passive piece is circumferentially fixed by the external spline and the internal spline of the clutch. The primary and passive sheets are in a cooling lubricant enclosed within the housing. When the clutch is in operation, the input shaft and the active piece connected thereto rotate, and the main and passive parts are pressed against each other by the hydraulic pressure to generate a friction torque, and the two synchronously rotate to realize power transmission. A section of a wet multiplate clutch is shown in Figure 4.

In the wet multiplate clutch, the normal state of the friction plate determines the key to whether the clutch can achieve its function. This study used the wet friction plate in the hydraulic control wet multiplate clutch as the research object and made a time censored test under normal working conditions. The test data are shown in Table 2.

From the number of failed samples in Table 2 and the actual project, the $p_{i}$ upper limit $p_{\text {upp }} \leq 0.5$, so let $p_{\text {upp }}=0.5$. The combination of (11) and (20) was used to calculate the each censoring time estimation of failure probability as $\widehat{p}_{i}(i=1,2, \ldots, 4)$, where $c=5$. The calculation results are shown in Table 3.

Putting the data in Tables 2 and 3 into (23), $\widehat{m}=2.0985$ and $\widehat{\eta}=2683.4$ were calculated; the calculation

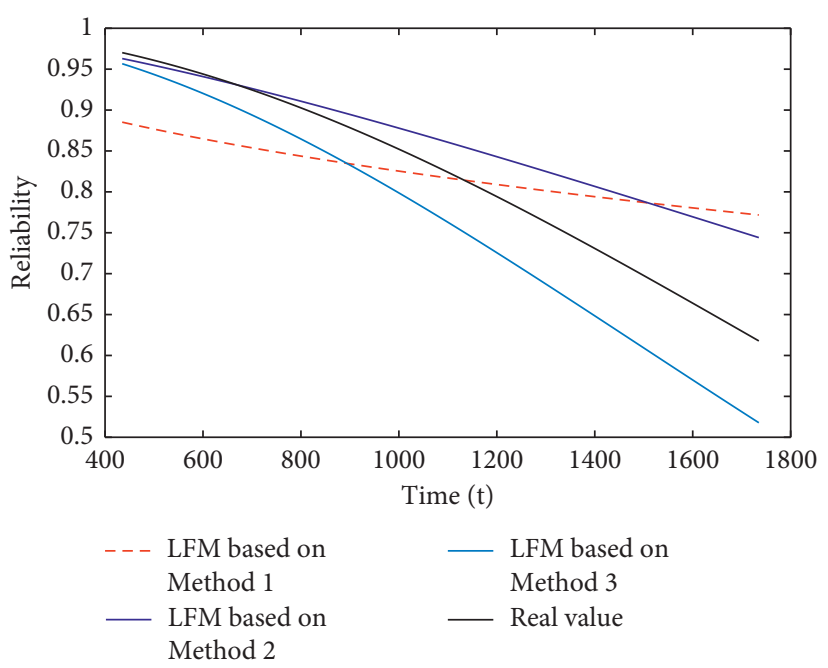

FIgURE 3: Reliability confidence lower limit comparison.

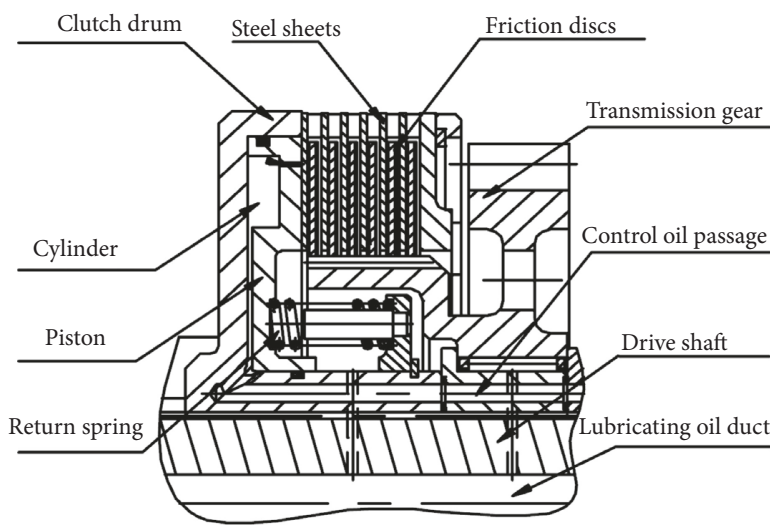

Figure 4: Sectional view of the integrated transmission clutch.

TABLe 2: Wet friction plate test data.

\begin{tabular}{lcccc}
\hline Subgroup number $i$ & 1 & 2 & 3 & 4 \\
\hline Censoring time $\tau_{i} / t$ & 500 & 1000 & 1500 & 2000 \\
Test samples $n_{i}$ & 3 & 3 & 3 & 3 \\
Remaining work samples $s_{i}$ & 12 & 9 & 5 & 3 \\
Failure samples $r_{i}$ & 0 & 0 & 1 & 0 \\
\hline
\end{tabular}

result was substituted into (24) and the point estimation curve of reliability under the Weibull distribution was obtained. It is shown in Figure 5.

This study sets the reliability $R$ at the confidence level $(1-\alpha)=0.7$, using (27), to obtain the point estimation of the upper bound $\hat{p}_{i}^{u}$ of the failure probability $p_{i}$. They are shown in Table 4.

By $\hat{y}_{i}^{u}=\ln \left[-\ln \left(1-\hat{p}_{i}^{u}\right)\right]$ and $x_{i}=\ln t_{i}, \hat{y}_{i}^{u}$ and $x_{i}$, were obtained. They are shown in Table 5 .

With (30) and (31), the lower confidence limit at any time $t$ can be obtained when reliability $R$ is at a confidence level of 0.7. It is shown in Figure 6.

With (33), the upper confidence limit of the failure rate function $\lambda_{u}(t)$ is obtained. It is shown in Figure 7. 
TABLE 3: Each censoring time $t_{i}$ point estimation value of failure probability point $\widehat{p}_{i}$.

\begin{tabular}{lcccc}
\hline Subgroup number $i$ & 1 & 2 & 3 & 4 \\
\hline Failure probability point estimation $\widehat{p}_{i}$ & 0.0256127 & 0.108449 & 0.258006 & 0.391425
\end{tabular}

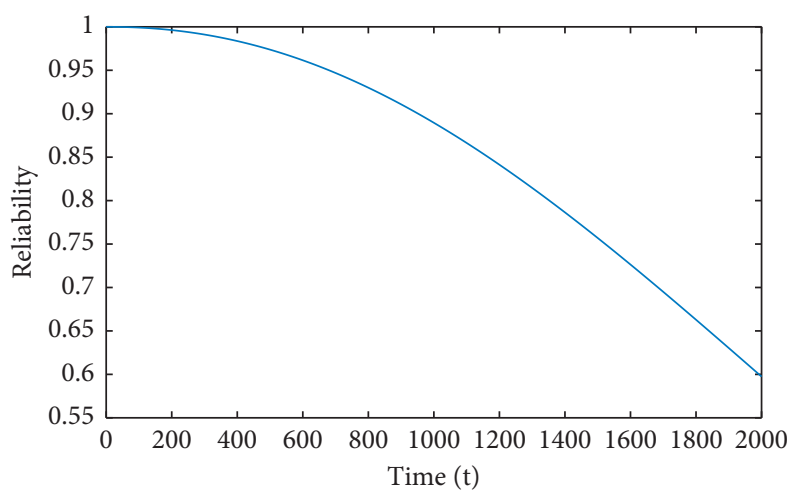

Figure 5: Reliability estimation curve.

TABLE 4: Failure probability $p_{i}$ confidence upper limit point estimation.

\begin{tabular}{lccc}
\hline Subgroup number $i$ & 1 & 2 & 3 \\
\hline $\begin{array}{l}\text { Failure probability confidence upper bound point } \\
\text { estimation } \widehat{p}_{i}^{u}\end{array}$ & 0.0259 & 0.1205 & 0.2890 \\
\hline
\end{tabular}

TABLE 5: Transformed values $\hat{y}_{i}^{u}$.

\begin{tabular}{lcccc}
\hline Subgroup number $i$ & 1 & 2 & 3 & 4 \\
\hline$\hat{y}_{i}^{u}$ & -3.6404 & -2.0526 & -1.0756 & -0.6249 \\
$x_{i}$ & 6.2146 & 6.9078 & 7.3132 & 7.6009 \\
\hline
\end{tabular}

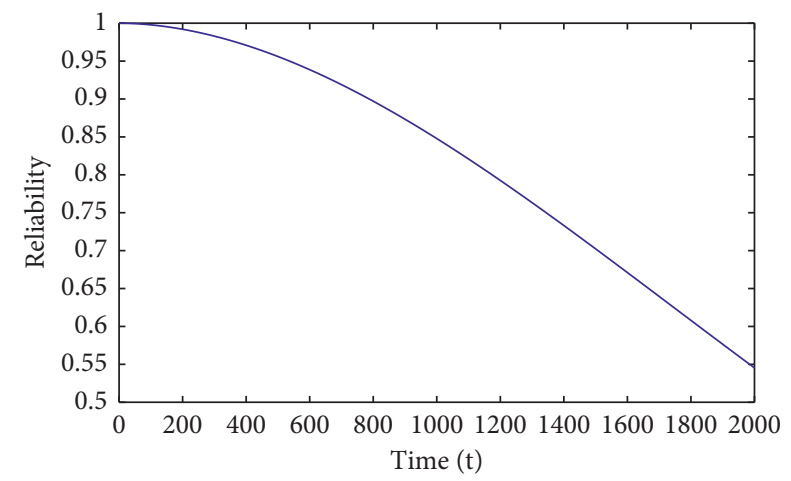

FIGURE 6: Reliability confidence lower limit estimation curve.

\section{Conclusion}

(1) In the case of the Weibull distribution, based on the time-censored test with very little failure data, the idea of the match distribution curve method combined with Bayesian theory and subtraction function method was used to study the reliability evaluation method, derived reliability parameter point estimation, and confidence interval estimation methods.

(2) According to the different values of $m$ in the Weibull distribution, the concave and convex properties of

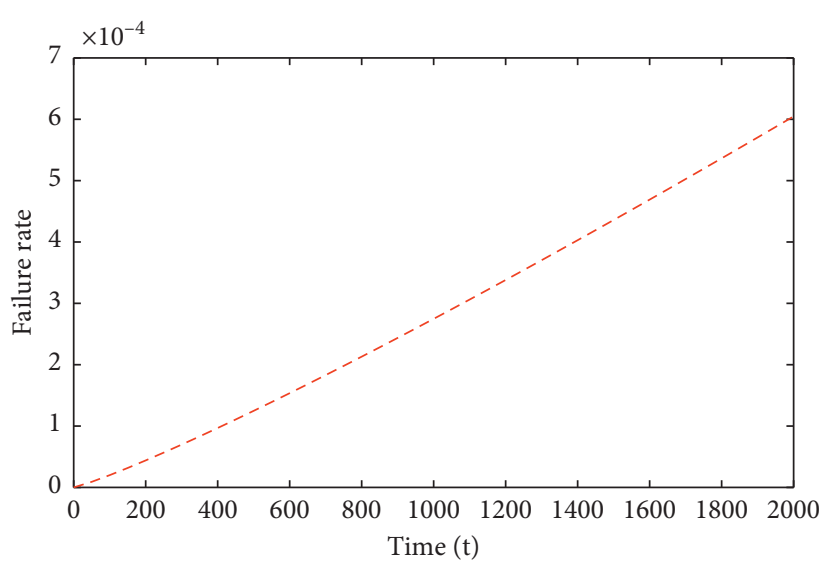

FIgURE 7: Failure rate confidence upper limit point estimation curve.

the distribution function and the function characteristics were used to determine the range of the failure probability. Combined with the reduction function of the failure probability, the parameters of the pretest distribution function were obtained. The value range further used the Bayesian method to obtain the point estimation failure probability $p_{i}$ based on the Bayesian poster distribution. 
(3) In view of the problem that the results of the reliability parameter estimation and the confidence interval estimation are "disjointed" in the current research, this study used the distribution curve method to unify the estimation method and avoid the "disjoint" phenomenon. The generation of the results and the comparison of the calculation results in the example further enhance the credibility of the results.

(4) Applying the method proposed in this study to the data analysis of a time-censored test of the wet friction plate in a certain type of hydraulic control wet multiplate clutch, the applicability of the proposed method was further verified.

\section{Data Availability}

The data used to support the findings of this study are included within the article.

\section{Conflicts of Interest}

The authors declare that they have no conflicts of interest.

\section{Acknowledgments}

This research was supported by the projects of the National Natural Science Foundation of China (grant nos. 71371183 and 71071158).

\section{References}

[1] Y. Kwon, "Design of Bayesian zero-failure reliability demonstration test for products with Weibull lifetime distribution," Journal of Applied Reliability, vol. 14, no. 4, pp. 220-224, 2014.

[2] H. Li, F. Chen, Z. Yang et al., Bayesian Reliability Assessment Method for Single NC Machine Tool under Zero Failures. Applications and Techniques in Information Security, Springer, Heidelberg, Germany, 2015.

[3] H. F. Martz and R. A. Waller, "A bayesian zero-failure (BAZE) reliability demonstration testing procedure," Journal of Quality Technology, vol. 11, no. 3, pp. 128-138, 1979.

[4] Q. John and M. R. Estimating, "The probability of rare events: addressing zero failure date," Risk Analysis, vol. 31, no. 7, pp. 1120-1132, 2011.

[5] X. Jia, P. Jiang, and B. Guo, "The estimation to failure probability for zero-failure data in Weibull distribution," Journal of Mechanical Strength, vol. 37, no. 2, pp. 288-294, 2015.

[6] D. Kurz, H. Lewitschnig, and J. Pilz, "Advanced bauesian estimation of Weibull early life failure distributions," Quality and Reliability Engineering International, vol. 30, no. 3, pp. 363-373, 2014.

[7] P. Jiang, Y. Xing, X. Jia, and B. Guo, "Weibull failure probability estimation based on zero-failure data," Mathematical Problems in Engineering, vol. 2015, Article ID 681232, 8 pages, 2015.

[8] Y.-C. Yin, H.-Z. Hong, W. Peng, Y.-F. Li, and J. Mi, "An E-bayesian method for reliability analysis of exponentially distributed products with zero-failure date," Maintenance and Reliability, vol. 18, no. 3, pp. 445-449, 2016.
[9] Y.-N. Kan, Z.-J. Yang, G.-F. Li, J.-L. He, Y.-K. Wang, and H.-Z. Li, "Bayesian zero-failure reliability modeling and assessment method for multiple numerical control (NC) machine tools," Journal of Central South University, vol. 23, no. 11, pp. 2858-2866, 2016.

[10] J. D. Chen, W. L. Sun, and B. X. Li, "On the confidence limits in the case of no failure data," Acta Mathematicae Applicatae Sinica, vol. 18, no. 1, pp. 90-100, 1995.

[11] H. Li, L. Xie, M. Li, J. Ren, B. Zhao, and S. Zhang, "Reliability assessment of high-quality and long-life products based on zero-failure data," Quality and Reliability Engineering International, vol. 35, no. 1, pp. 470-482, 2019.

[12] X. Jia, The Estimation of Reliability for Satellite Platform with Multiply Type-I Censored Data, National University of Defence Technology, Changsha, China, 2017.

[13] T.-Q. Xu and Y.-P. Chen, "Two-sided M-bayesian credible limits of reliability parameters in the case of zero-failure data for exponential distribution," Applied Mathematical Modelling, vol. 38, no. 9-10, pp. 2586-2600, 2014.

[14] K. Cheng, Life Distribution Class and Reliability Mathematical Theory, Science Press, Beijing, China, 1999.

[15] X. Jia, X. Wang, and B. Guo, "Reliability assessment for very few failure data and zero-failure data," Journal of Mechanical Engineering, vol. 52, no. 2, pp. 182-188, 2016.

[16] Y.-J. Lin and Y. L. Lio, "Bayesian inference under progressive type-I interval censoring," Journal of Applied Statistics, vol. 39, no. 8, pp. 1811-1824, 2012.

[17] J. K. Zhang and X. M. Tang, Bayes Methods, National Defense Science and Technology University Press, Changsha, China, 1989.

[18] S. S. Mao, J. F. Xia, and W. Q. Guan, "The reliability analysis of zero-failure data for bearing life test," Chinese Journey of Applied Probability and Statistics, vol. 9, no. 3, pp. 326-331, 1993.

[19] M. Han, "Confidence limits of reliability parameters for Weibull distribution," Journal of Mechanical Strength, vol. 31, no. 1, pp. 59-62, 2009.

[20] L. F. Zhang, M. Xie, and L. C. Tang, "A study of two estimation approaches for parameter of Weibull distribution based on WPP," Reliability Engineering and System Safety, vol. 92, no. 3, pp. 360-368, 2007. 


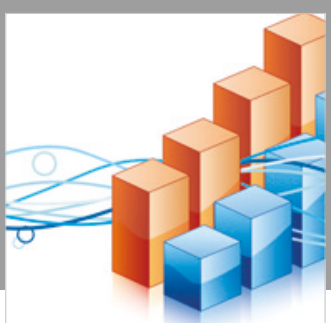

Advances in

Operations Research

\section{-n-m}
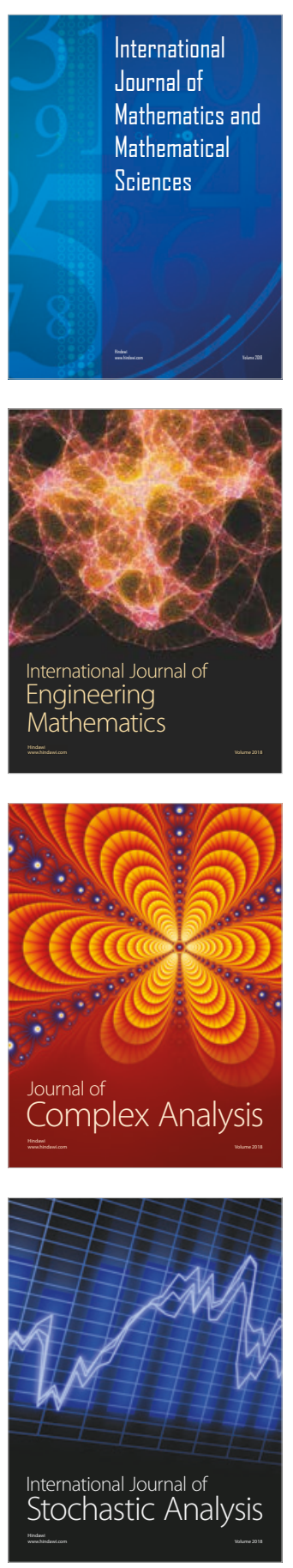
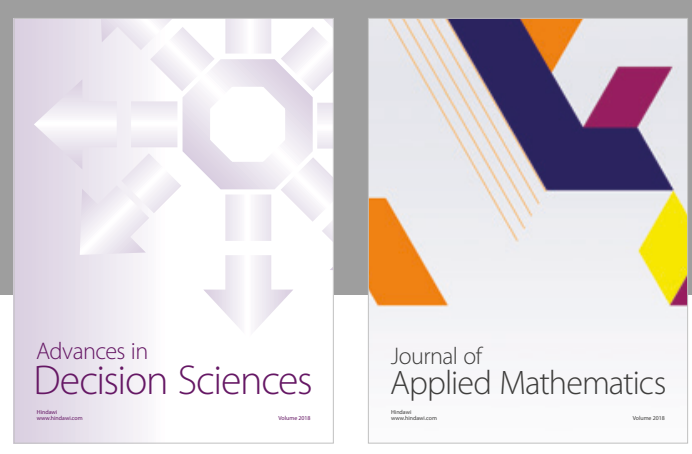

Journal of

Applied Mathematics
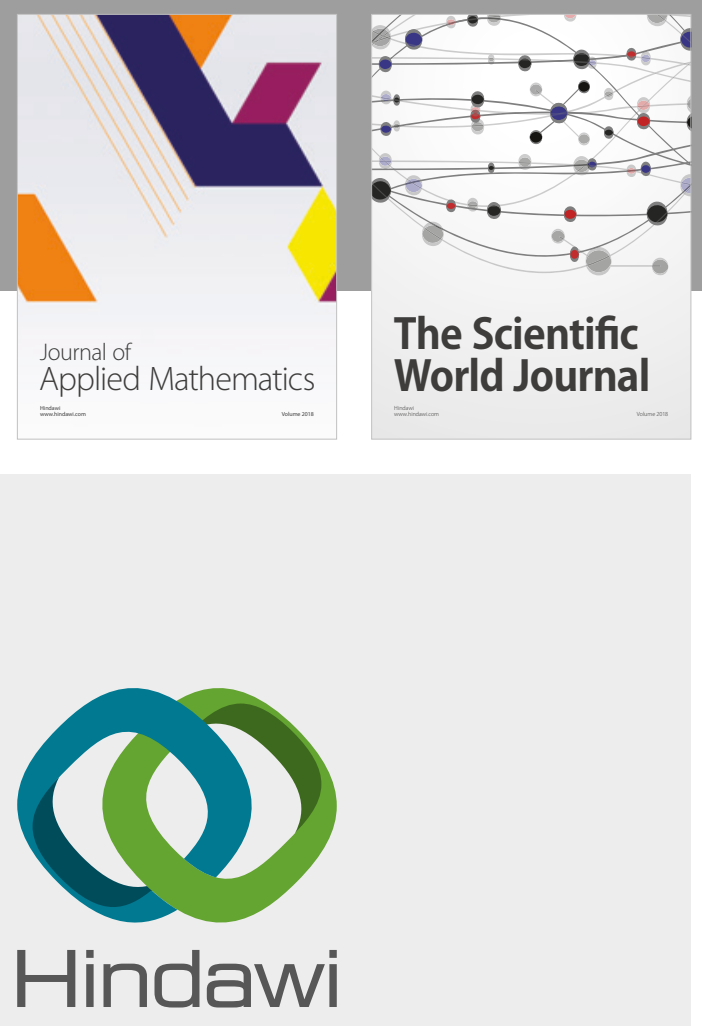

Submit your manuscripts at

www.hindawi.com

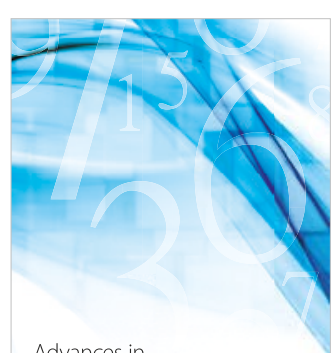

Advances in
Numerical Analysis
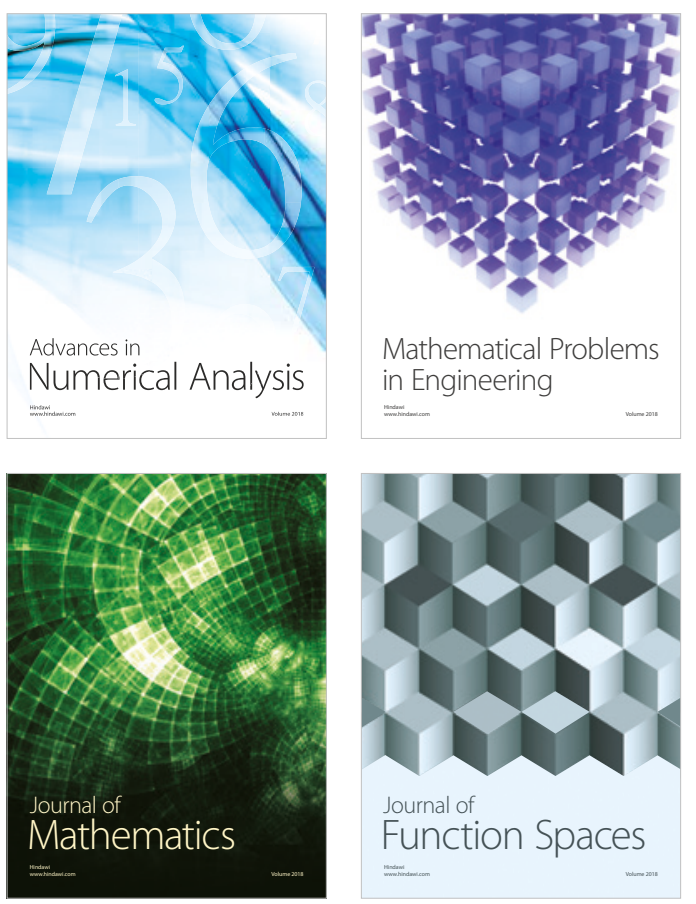

Mathematical Problems in Engineering

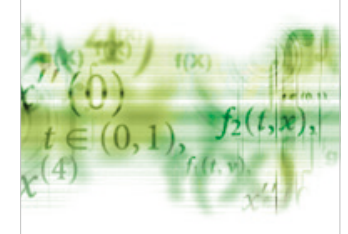

International Journal of

Differential Equations

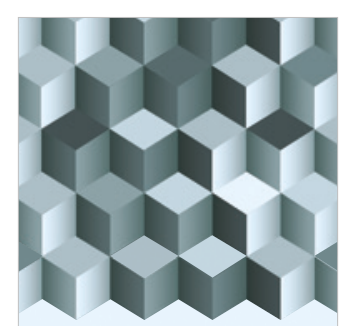

Journal of

Function Spaces

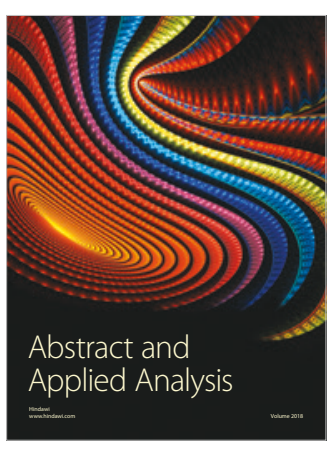

The Scientific

World Journal

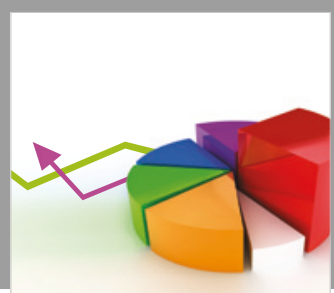

Journal of

Probability and Statistics
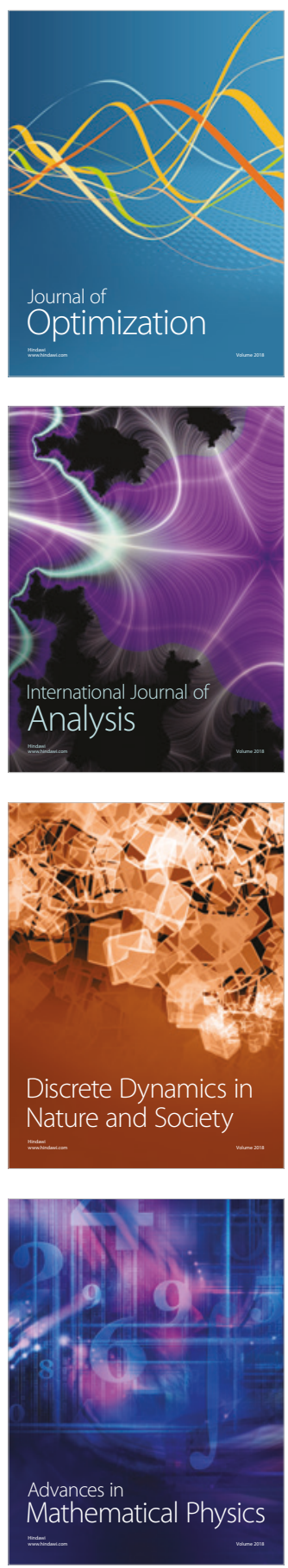